\title{
Article
}

\section{Génie Chimique}

\author{
Aymen LABIDI $1,+, \neq$ (i)
}

Citation: LABIDI, A Génie Chimique . Chemical Engineering 2021, 1, 0. https://doi.org/

Published: $6^{\text {th }}$, February2021

Author's Note: You can Visit my Researchgate and Linkedin profiles for more researches.

Copyright: (C) 2021 by the author. Licensee to Aymen Labidi for Chemical Engineering - Open Resource (CC BY) license (https://creativecommons.org/licenses/by/4.0/).
1 Affiliation: University of Carthage - National Institute of Applied Sciences and Technology - Chemical Engineering Student - Chemical Engineering Department

+ Current address: 676 INSAT Centre Urbain Nord BP Tunis Cedex 1080

$\ddagger$ Aeronautical Consultant for FTAA; Glider Former and Manager for Aerobotix INSAT .

\begin{abstract}
:
Le génie chimique, ou génie des procédés physico-chimiques, désigne l'application de la chimie physique à l'échelle industrielle. Elle a pour but la transformation de la matière dans un cadre industriel et consiste en la conception, le dimensionnement et le fonctionnement d'un procédé comportant une ou plusieurs transformations chimiques et/ou physiques. Les méthodes utilisées dans un laboratoire ne sont souvent pas adaptées à la production industrielle d'un point de vue économique et technique. Le génie chimique permet ainsi le passage d'une synthèse de laboratoire à un procédé industriel de même que son fonctionnement dans le respect des contraintes économiques, techniques, environnementales et de sécurité.[1] Dans ce résumé de cours, vous allez trouvez l'essentiel du génie chimique pour un débutant (à l'attention des étudiants de la Deuxiéme année chimie industrielle - INSAT) ainsi que les astuces de cours pour assurer et une bonne compréhension et une bonne maîtrise de cette discipline.
\end{abstract}

Keywords: Thermodynamique, Diagrammes Thermodynamiques, Génie Climatique

\section{Rappel de Thermodynamique}

\section{Processus Isotherme}

Un processus isotherme est en thermodynamique une transformation chimique ou physique d'un système au cours de laquelle la température du système est constante et uniforme.

\section{Processus Isobare}

En thermodynamique, un processus isobare est une transformation chimique ou physique d'un système au cours de laquelle la pression du système reste constante et uniforme. La pression d'un tel système est définie, ce qui implique que la transformation est quasistatique. Un processus isobare diffère $d^{\prime}$ un processus monobare dans lequel la pression peut temporairement varier.

\section{Processus Isochore}

En thermodynamique, un processus isochore est une transformation chimique ou physique d'un système au cours de laquelle le volume du système est constant.

\section{Processus Isentropique}

En thermodynamique, un processus isentropique est un processus thermodynamique au cours duquel l'entropie du système étudié reste constante. La constance de l'entropie peut être obtenue par un processus idéal qui est à la fois adiabatique et réversible.

\section{Processus Polytropique}

Une transformation polytropique est une modification de l'état thermodynamique d'un système avec un échange thermique partiel entre ce dernier et son environnement. Un état polytropique se classe donc entre le cas isotherme et le cas adiabatique.

\section{Fluide Compressible}

Un fluide est dit compressible si son volume varie quand sa pression change. Débit massique à l'entrée = Débit massique à la sortie

$$
q_{m e}=q_{m s}
$$




\section{Fluide Incompressible}

Un fluide incompressible est un fluide dont le volume est considéré comme constant quelle que soit la pression qu'il subit, tout fluide étant en réalité sensible à la pression. On exprime ainsi son débit volumique selon cette égalité

$$
q_{v e}=q_{v s}
$$

Néanmoins, attention aux rétrécissements et élargissements des sections d'entrée et de sortie.

\section{Régime Permanent}

Un régime d'écoulement est dit permanent ou stationnaire si les paramétres qui le caractérisent (pression, température, vitesse, pression...) ont une valeur constante au cours du temps, c'est à dire indépendante du temps.

En régime stationnaire, le débit massique est le même à travers toutes les sections droites d'un même tube de courant

Le Débit volumique s'exprime en fonction de la vitesse d'écoulement du fluide dans la conduite et la section de celle-ci:

$$
q_{v}=V S
$$

\section{$\mathbf{1}^{e r}$ principe de la Thermodynamique}

\section{Enoncé du premier principe}

Lorsqu'un systéme passe d'un état initial à un état final, la somme du travail et de la chaleur échangés est indépendante du chemin suivi. soit U l'énergie interne:

$$
d U=\delta Q+\delta W
$$

et

$$
\Delta U=Q+W
$$

U est une fonction d'état.

\section{Notion d'Enthalpie}

$\mathrm{U}$ et $\mathrm{pV}$ combinent une fonction d'état. On l'appelle enthalpie et on la désigne par la lettre $\mathrm{H}$

$$
H=U+p V
$$

\section{Relation de Mayer}

On peut montrer que

$$
C_{p}-C_{v}=R
$$

R étant la Constante du gaz parfait

$$
C_{v}=\frac{3}{2} R
$$

Pour les gaz parfaits monoatomiques

$$
C_{v}=\frac{5}{2} R
$$

Pour les gaz parfaits diatomiques

\section{La loi de Joule}

L'énergie interne et l'enthalpie d'un gaz parfait ne dépendent que de la témpérature, à une température constante $\mathrm{T}$ :

$$
\Delta U=\Delta H=0
$$

La Loi de Laplace

En thermodynamique, la loi de Laplace est une relation reliant la pression et le volume d'un gaz parfait subissant une transformation isentropique (ou adiabatique et réversible) [2] [3] 


$$
\begin{gathered}
P V^{\gamma}=C t e \\
T V^{\gamma-1}=C t e \\
T^{\gamma} P^{1-\gamma}=C t e
\end{gathered}
$$

Remarque: le premier principe de la Thermodynamique des systèmes ouverts en régime stationnaire

$$
\Delta H+\Delta \frac{1}{2} m V^{2}+\Delta m h z=\sum W+\sum Q
$$

le premier principe de la Thermodynamique des systèmes fermés en régime stationnaire

$$
\Delta U+\Delta E_{c}+\Delta E_{p}=\sum W+\sum Q
$$

$2^{\text {nd }}$ principe de la Thermodynamique

Enoncé du Second principe

Pour tout systéme thermodynamique, il existe une fonction d'état notée $S$, appelée entropie, dont la variation au cours d'une transformation quelconque l'amenant d'un état 1 à un état 2 est la somme de deux termes:

$$
S_{2}-S_{1}=\Delta S=\Delta S_{e}+\Delta S_{i}
$$

$\Delta S_{e}$ est la variation d'entropie résultant de l'échange de chaleur entre le système et le milieu extérieur. Cette quantité qui peut être positive, négative ou nulle, a pour expression:

$$
\Delta S_{e}=\int_{1}^{2} \frac{\delta Q}{T_{S}}
$$

$\Delta Q$ étant la quantité de chaleur "élémentaire" échangée entre le système et la source de chaleur responsable de cet échange et dont la tempéature est $T_{s} . \Delta S_{i}$ est l'entropie créée à l'intérieur du système au cours de la transformation:

$$
\Delta S_{i}=\Delta S-\int_{1}^{2} \frac{\delta Q}{T_{S}}
$$

$\Delta S_{i}$ est strictement positive pour tout le processus réél (donc irréversible) et nulle dans le cas de la réversibilité. Cherchons la variation de l'entropie de l'univers lors d'une transformation quelconque:

$$
\Delta S_{\text {univers }}=\Delta S_{\text {systme }}+\Delta S_{\text {milieuextrieur }}
$$

Finalement:

Pour une transformation réversible $\Delta S_{\text {Univers }}=0$

Pour une transformation irréversible $\Delta S_{\text {Univers }}>0$

$3^{e}$ principe de la Thermodynamique

La Température de la zéro absolue est inaccessible.

L'entropie d'un corps pur cristallisé est nulle au zéro absolue: $S=0$ à $0 \mathrm{~K}$

Consultez ce lien vers mon profil researchgate pour voir le tableau résumant toutes les Transformations thermodynamiques de façon détailée 


\section{Machine Thermique -Les Cycles Thermodynamiques}

\section{Machine Thermique}

Une Machine Thermique est un dispositif dans lequel le fluide décrit un cycle de transformation, une machine thermique est dite ditherme si elle échnage de l'énergie par transfert thermique avecdeux sources de chaleur: source froide et chaude.

Pour une machine ditherme, les échanges d'énergies par transfert thermique sont tels que:

$$
\frac{Q_{c}}{T_{e}}+\frac{Q_{f}}{T_{f}} \leq 0
$$

$\rightarrow$ c'est l'inégalité de Clausius Clapeyron

\section{Cycle Thermodynamique}

Un cycle thermodynamique est une suite de transformations successives qui part d'un système thermodynamique dans un état donné, le transforme et le ramène finalement à son état initial, de manière à pouvoir recommencer le cycle. Au cours du cycle, le système voit sa température, sa pression ou d'autres paramètres d'état varier, tandis qu'il échange du travail et réalise un transfert thermique avec l'extérieur.

Le cycle est dit Moteur quand il s'agit du cycle le plus efficace pour obtenir du travail à partir de deux sources de chaleur de températures constantes comme des thermostats, il est décrit dans le sens anti-trigonométrique, sens des aiguilles d'un montre, sens direct. Par contre, le cycle est dit Résistant ou récepteur quand il s'agit du moyen le plus efficace pour transferer de la chaleur d'une source chaude à une source froide à partir d'une source de travail, il est décrit dans le sens inverse.

\section{Efficacité - Rendement}

Le rendement noté $\eta$ est le rapport du transfert d'énergie utile au transfert d'énergie depensée pour le fonctionnement.

\section{Enoncé de Kelvin}

Il n'existe pas de moteurs fonctionnant de maniére cyclique qui produisent du travail à partir d'une seule source de chaleur.

\section{Enoncé de Clausius}

Il n'existe pas de processus dont le seul effet serait de faire passer de la chaleur d'une source foide à une source chaude.

\section{Machine de Carnot}

Carnot a montré que la transformation de chaleur en travail n'est possible que si l'on dispose de 2 sources de chaleurs à 2 temps différents.

\section{Cycle de Carnot}

Le cycle de Carnot est un cycle thermodynamique théorique pour un moteur ditherme, constitué de quatre processus réversibles : une détente isotherme réversible, une détente adiabatique réversible (donc isentropique), une compression isotherme réversible, et une compression adiabatique réversible. 


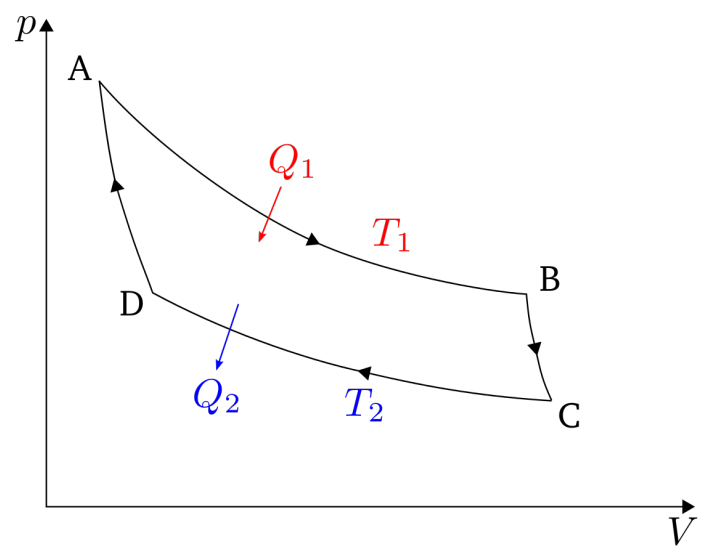

Figure 1. Cycle de Carnot moteur dans le Diagramme de Clapeyron - $\mathrm{AB}$ : détente isotherme ; $\mathrm{BC}$ : détente adiabatique ; $\mathrm{CD}$ : compression isotherme ; - DA : compression adiabatique. .

Le rendement Thermodynamique du cycle de Carnot est

$$
r_{\xi}=\frac{-W_{\text {Cycle }}}{Q_{c}}=1-\frac{T_{f}}{T_{c}}
$$

avec $\mathrm{W}<0 ; Q_{c}>0$ et $Q_{f}<0$

\section{Machine Frigorifique}

La fonction d'une machine frigorifique est de prendre de la chaleur du côté basse température en produisant une évaporation du fluide frigorigéne qui se condense par la suite sur la paroi du fond de l'appareil et de le rejeter à l'exterieur grâce à la grille située derriére l'appareil en utilisant une énergie externe pour entretenir le processus.

On va transformer la chaleur de la partie à refroidir vers la partie qui sera réchauffée

Pompe à Chaleur

Une pompe à Chaleur (PAC) appelée aussi Thermolyse est un dispositif permettant de transférer de l'énergie thermique (calories) d'un milieu à basse température vers un milieu à haute température.

\begin{tabular}{lcc}
\hline & Efficacité & Efficacité de Carnot \\
\hline Moteur & $\frac{-W}{Q_{c}}$ & $1-\frac{T_{f}}{T_{c}}$ \\
Réfrigérateur & $\frac{Q_{f}}{W}$ & $\frac{T_{f}}{T_{c}-T_{f}}$ \\
Pompe à chaleur & $\frac{-Q_{c}}{W}$ & $\frac{T_{c}}{T_{c}-T_{f}}$ \\
\hline
\end{tabular}

Table 1: Tableau Récapitulatif des Coefficients de Performances

\section{Attention!!}

Pour passer des quantités de chaleur aux températures dans une machine frigo/PAC/Moteur, on utilise l'inégalité de Clausius Clapeyron.

Si la température intérieure d'un réfrigérateur varie au cours du temps, on utilise la relation de Clausius (égalité ou inégalité) selon lemode de fonctionnement 


\section{Diagramme de Raveau}

Le diagramme de Raveau est un diagramme thermodynamique qui permet d'interpréter le travail d'une mahcine thermique ditherme en fonction de ses transferts thermiques avec sa source chaude et source froide.

\section{Zone I}

C'est le domaine des cycles moteurs: La machine fournit du travail en prenant de la chaleur à la source chaude et en en cédant à la source froide. C'est donc un moteur thermique. $Q_{c}>0 ; Q_{f}<0 ; W<0$

\section{Zone II}

La machine consomme du travail en prenant de la chaleur à la source chaude et en en cédant à la source froide. Ces machines sont peu intéressantes car on peut obtenir la même chose sans consommer de travail. $Q_{c}>0 ; Q_{f}<0 ; W>0$

\section{Zone III}

La machine consomme du travail en cédant de la chaleur aux deux sources. Elle est peu intéressante et se rapproche des machines monothermes. $Q_{c}<0 ; Q_{f}<0 ; W>0$

\section{Zone IV}

La machine consomme du travail et transfert de l'énergie thermique de la source froide à la source chaude, le domaine des pompes à chaleur et machines frigorifiques $Q_{c}<0$; $Q_{f}>0 ; W>0$

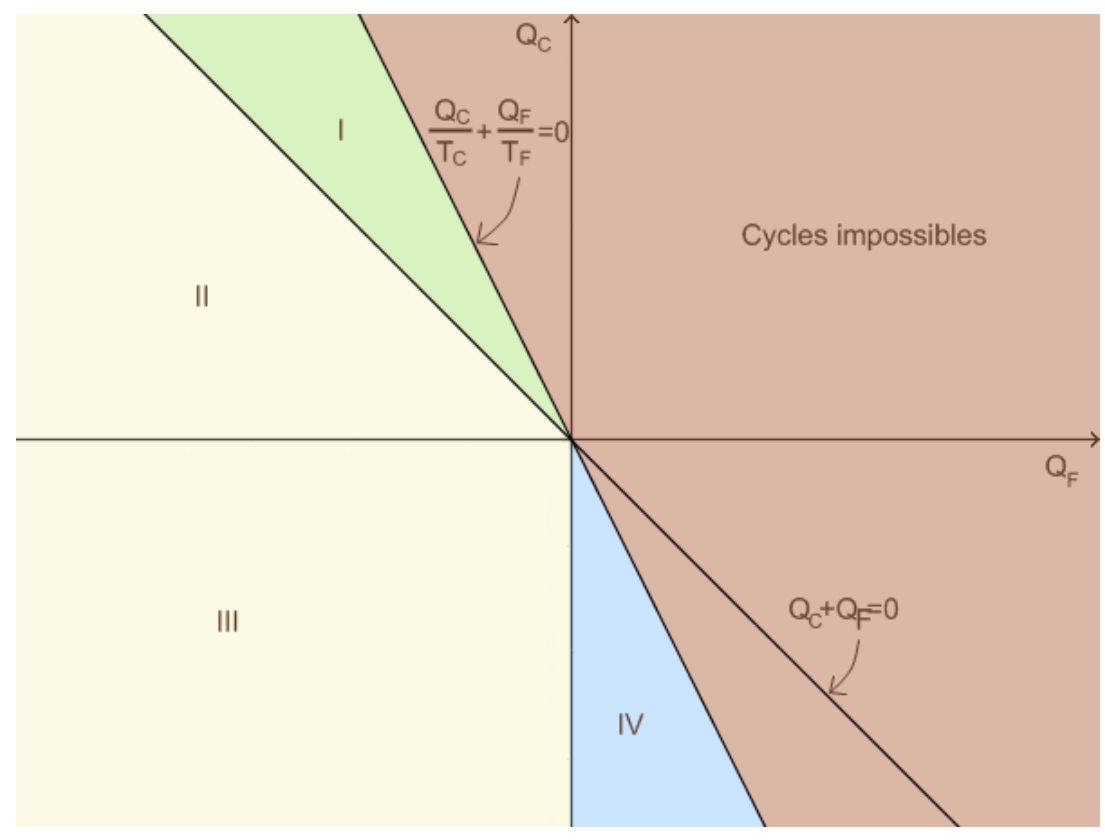

Figure 2. Diagramme de Raveau

\section{Moteurs à combustion interne}

Le moteur à combustion interne $(\mathrm{MCI})$ est un type de moteur à combustion dans lequel le combustible est brûlé dans le cylindre d'une machine à piston.

L'énergie thermique dégagée par la combustion est convertie en energie mécanique à l'intérieur du moteur.

\section{Cycle de Beau de Rochas}

Le cycle de Beau de Rochas, cycle à quatre temps ou encore cycle d'Otto est un cycle thermodynamique théorique. Son principal intérêt pratique réside dans le fait que les moteurs à explosion à allumage commandé, généralement des moteurs à essence tels que ceux utilisés dans les automobiles, ont un cycle thermodynamique pratique qui peut être représenté de manière approchée par le cycle de Beau de Rochas.

Le cycle peut être modélisée de la maniére suivante: 
- l'admission est modélisée par une détente isobare (0-1),

- la compression est supposée adiabatique (1-2),

- la détente est adiabatique (3-4),

- $\quad$ l'ouverture de la soupape est modélisée par une détente isochore (4-5)

- l'échappement est modélisé par une détente isobare (5-0)

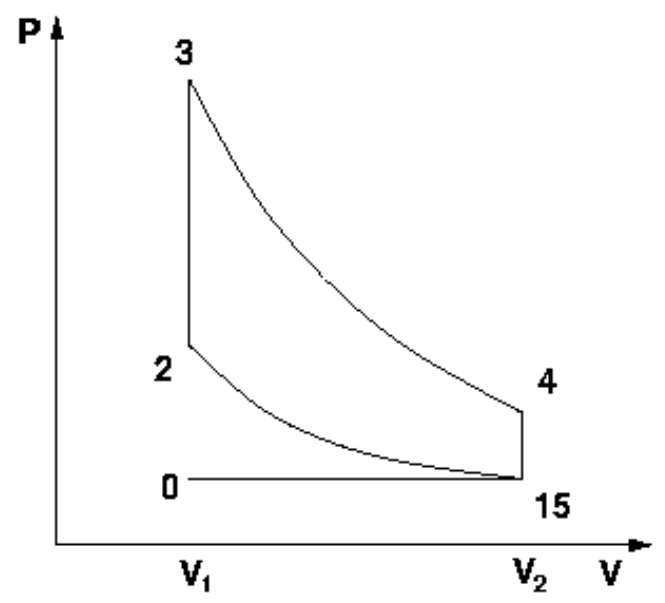

Figure 3. Représentation du cycle de Beau de Rochas sur le diagramme de Clapeyron (P,V)

Le rendement de ce cycle est donné par

$$
r=1-\frac{T_{4}-T_{1}}{T_{3}-T_{2}}
$$

En désignant par $a=\frac{V_{1}}{V_{2}}$ le taux de compression volumétrique, on peut exprimer le rendement autrement

$$
r=1-a^{(1-\gamma)}
$$

Remarque :

- Dans un moteur à essence, on fait monter en pression un mélange gazeux air/combustible quasiment homogéne puis on provoque son infallamation à l'aide d'une éteincelle commandée: un front de flamme se répand à partir de ce point d'ignition puis atteint progressivement tout le mélange, on évite précisément les situations d'auto-allumage car elles provoquent un phènomène de cliquetis, c'ést à dire que le combustion est anormale et peut donc entrainer un bruit métallique bien connu, et à terme des dégâts materiels, ils peuvent être graves.

- Si on nous demande d'exprimer le rendement en fonction d'un rapport de compression/détente volumétrique, on doit exprimer les températures du numérateur en fonction de celles du dénominateur moyennant les lois de Laplaces de transformations isentropiques

\section{Cycle Diesel}

Le moteur Diesel(Moteur à combustion à pression constante), appelé également moteur à allumage par compression, est un moteur à combustion et explosion dont la combustion est déclenchée lors de l'injection de carburant dans la chambre de combustion, par un phénomène d'auto-inflammation lié aux températures élevées dans la chambre de combustion. Celles-ci sont atteintes grâce à un fort taux de compression, permettant d'obtenir une température de 700 à $900^{\circ} \mathrm{C}$. Pour le démarrage des petits moteurs, des bougies de préchauffage sont souvent utilisées pour permettre un meilleur démarrage à froid, en créant un point chaud dans la chambre de combustion. Pour les moteurs de bateaux et les gros moteurs fixes à fioul lourd, on chauffe celui-ci à haute température pour permettre le démarrage. On peut modéliser le fonctionnement d'un tel moteur par le cycle suivant: 
- La Phase d'admission MA

- La Compression Adiabatique AB

- La Combustion s'effectuant à pression constante BC

- La Détente Adiabatique CD

- La Phase de refoulement/échappement DA

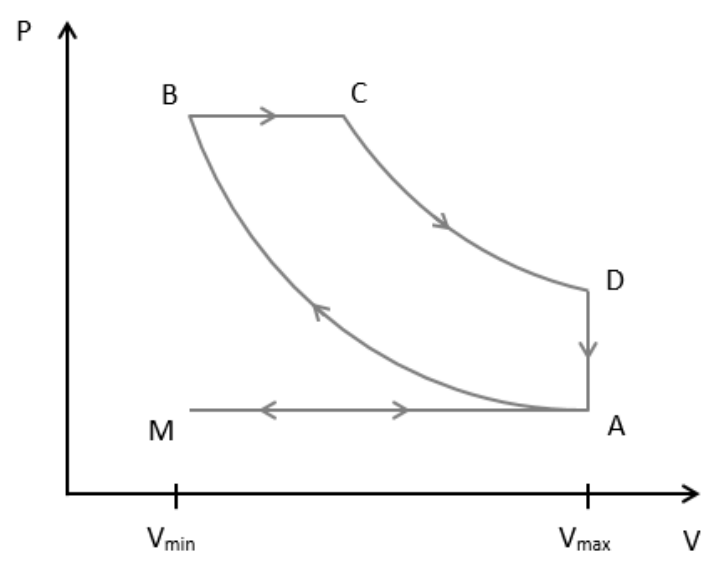

Figure 4. Diagramme pression-volume du cycle d'un moteur Diesel

On démontre que le rendement du cycle à pression constante s'exprime de la maniére suivante:

$$
r=1-\frac{T_{D}-T_{A}}{\gamma\left(T_{C}-T_{B}\right)}
$$

En désignant par $a=\frac{V_{A}}{V_{B}}$ le taux de compression volumétrique, et par $b=\frac{V_{D}}{V_{C}}$ le taux de détente volumétrique, on peut exprimer le rendement autrement

$$
r=1-\frac{a b^{(1-\gamma)}-b a^{(1-\gamma)}}{\gamma(a-b)}
$$

\section{Cycle de Sabathé}

Le cycle de Sabathé, appelé aussi cycle mixte de Sabathé, cycle de Trinkler ou cycle de Seiliger est un cycle de fonctionnement des moteurs thermiques. Il permet d'expliquer les cycles D'Otto/Beau de Rochas (moteurs à essence, combustion uniquement à volume constant) et Diesel (moteur diesel, combustion uniquement à pression constante). Ces deux exemples de cycles thermodynamiques sont en réalité des cas particuliers du cycle de Sabathé.

Ce cycle est dit mixte car il a particularité de diviser sa combustion en deux parties l'une à volume constant et l'autre à pression constante. Cette décomposition semble plus adaptée dans la réalité car elle permet une combustion complète et donc moins de rejets d'imbrûlés (monoxyde de carbone $\mathrm{CO}$ ou carburant non-utilisé).

Le cycle se compose de 5 transformations élémentaires[4] :

- $\quad$ compression isentropique, adiabatique reversible (1-2) ;

- combustion à volume constant, isochore (2-3) ;

- $\quad$ combustion à pression constante, isobare (3-4) ;

- détente isentropique, adiabatique reversible (4-5) ;

- refoulement à volume constant, isochore (5-1). 


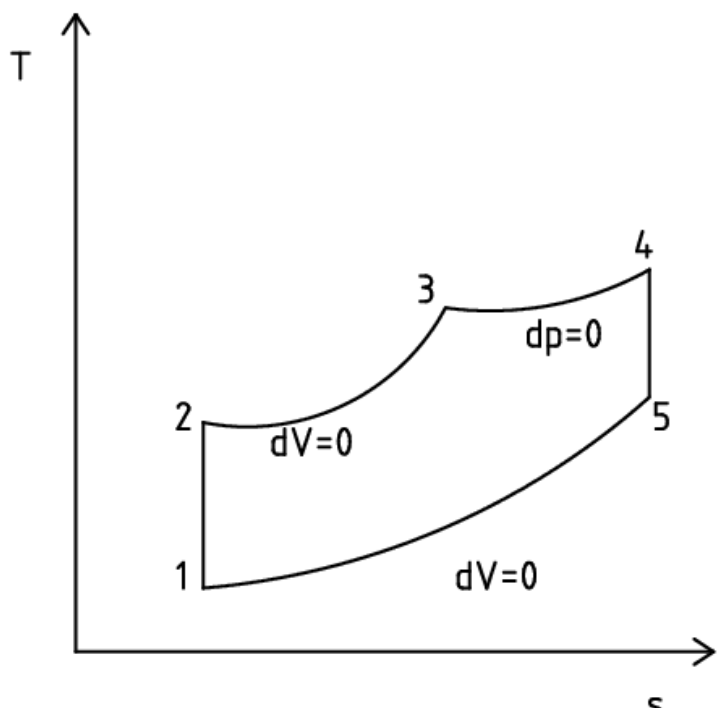

Figure 5. Cycle de Sabathé représenté sur un diagramme T,s (Température T et Entropie s)

\section{Cycle d'Atkinson}

Le cycle d'Atkinson est un cycle thermodynamique utilisé dans un moteur à combustion. Il a été inventé par James Atkinson en 1882. Ce cycle, qui utilise une détente plus grande que la compression, améliore le rendement au prix d'une puissance plus faible. Il est utilisé dans les voitures hybrides modernes[5].

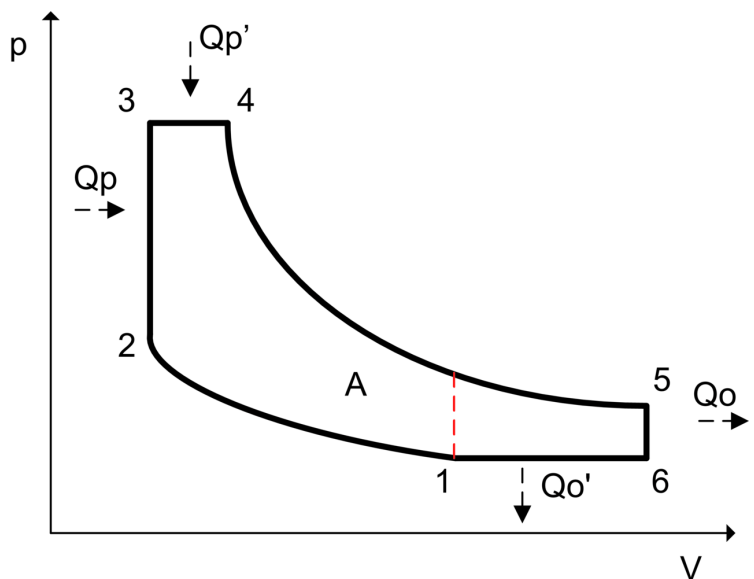

Figure 6. Cycle d'Atkinson

Le cycle d'Atkinson idéal est composé de :

- 1-2 compression adiabatique réversible ou isentropique ;

- 2-3 absorption de chaleur isochore, la pression augmente rapidement mais le piston ne bouge pas encore ;

- 3-4 absorption de chaleur isobare, la combustion continue et le piston se déplace ;

- 4-5 détente isentropique ;

- 5-6 dégagement de chaleur isochore ;

- 6-1 dégagement de chaleur isobare.

\section{Cycle de Rankine}

Le cycle de Rankine est un cycle thermodynamique endoréversible qui comprend deux isobares et deux adiabatiques.

C'est le cycle qui se rapproche le plus du cycle de Carnot. Il se distingue de ce dernier par la substitution de deux transformations isobares aux deux transformations isothermes, ce qui rend possible sa réalisation technique. 


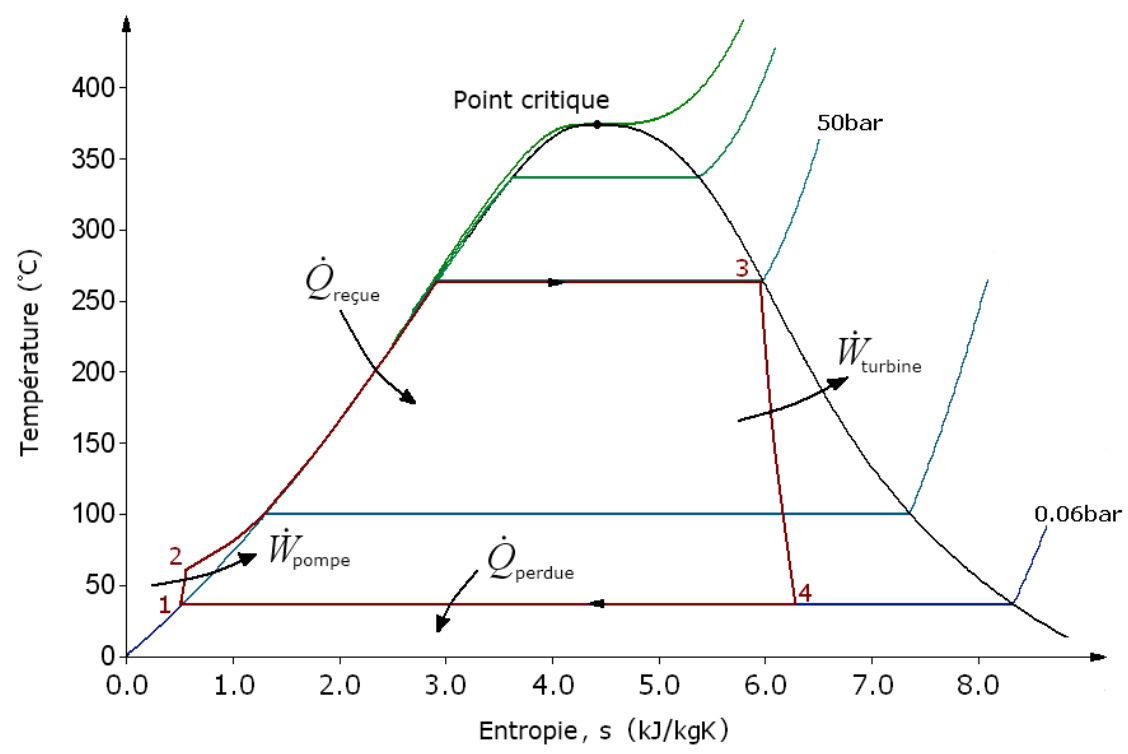

Figure 7. Diagramme entropique du cycle de Rankine avec l'eau comme fluide de travail

\section{Diagramme de Watt}

Le diagramme de Watt est un diagramme dans lequel on représente la pression d'un système thermodynamique en fonction de son volume.

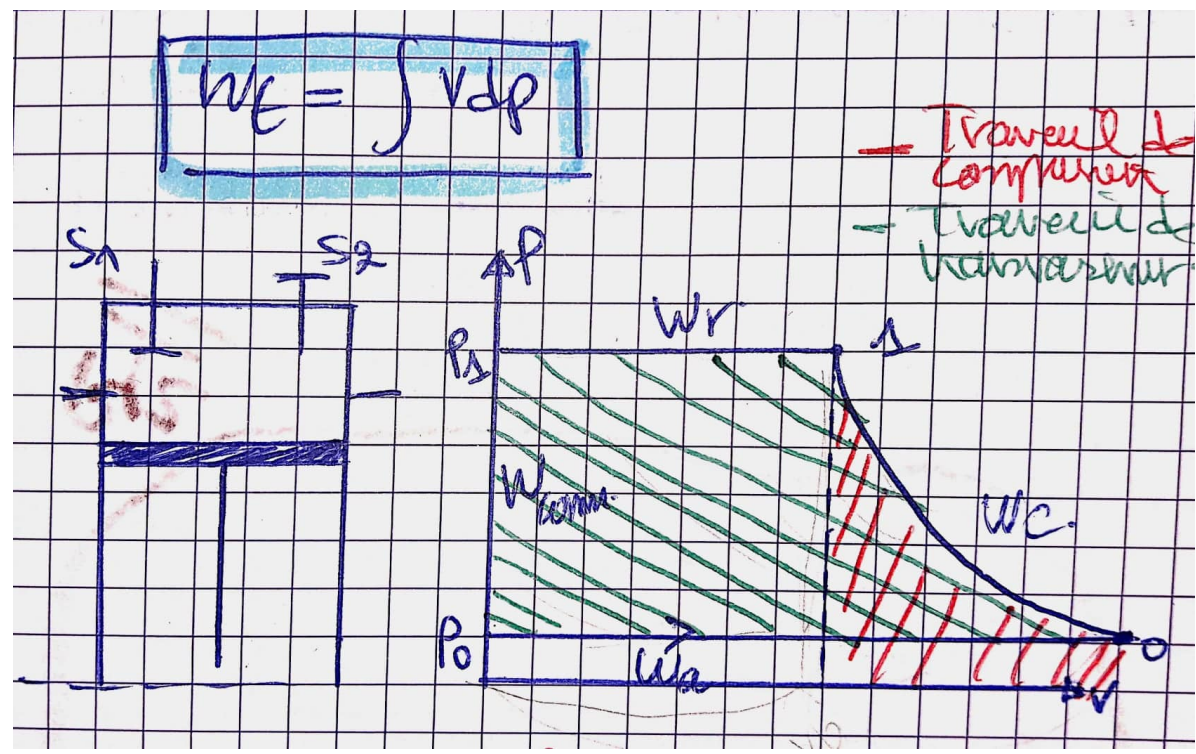

Figure 8. Diagramme de Watt indiquant les travaux de compression et de transvasement

C'est quoi le travail de Transvasement ? Le travail de transvasement intervient à partir du moment où on fait passer au travers d'une machine thermodynamique, un gaz de la pression P1 à la pression P2 ( tuyère, turbine, détente de JouleThomson ... )

- Transformation sans Transvasement (Surface Hachurée en rouge)

$$
W=-\int_{V 1}^{V 2} P d v
$$


- Trnsformation avec Transvasement (Surface Hachurée en Vert)

Initialement, les 2 soupapes sont fermées, on tire sur le piston, $S_{1}$ s'ouvre et l'air rétablit la pression à $P_{0}$ (Aspiration)

- Phase d'admission: La soupape d'admission s'ouvre, le piston se déplace au Point Mort Bas (PMB), le cylindre se remplit de gaz jusqu'à $V_{0}$, la masse du gaz croit à pression et température constantes.

- Phase de Compression: La soupape d'admission se ferme, le gaz sera comprimé suivant une transformation thermodynamique.

- Phase de refoulement: La soupape de refoulement s'ouvre, le gaz est refoulé à pression constante et partant la masse du gaz dans le cylindre va diminuer (étant donnée qu'il s'agit d'un système ouvert)

- Phase de Commutation: Il y'aura une chute instantannée de la pression à volume constant. Le piston atteint le fond du cylindre, $S_{2}$ se ferme et un nouveau cycle recommence à nouveau.

\section{Bilan Mécanique}

- $\quad$ Phase d'admission

$$
W_{0}=-P_{0} V_{0}
$$

- $\quad$ Phase de Compression

$$
W_{c}=-\int P d v
$$

- $\quad$ Phase de Refoulement

$$
W_{1}=P_{1} V_{1}
$$

- $\quad$ Phase de Commutation

$$
W=0
$$

D'où le travail Total sera $W_{t}=\int v d p$

Comment calculer ce Travail ?

Compression Isotherme:

$$
W_{\text {Transvasement }}=W_{\text {Compression }}=m r T \ln \left(\frac{P_{1}}{P_{0}}\right)
$$

Compression Isentropique:

$$
W_{\text {Transvasement }}=\gamma W_{\text {Compression }}
$$

\section{Remarques}

- En écrivant l'équation de la conservation de l'énergie, il faut obligatoirement l'exprimer de maniére générale.

- $\quad$ pour passer de l'énergie à la puissance, on divise par le temps

- Lors de l'interpolation linéaire, il faut prendre les deux valeurs de la pente sur la même table (pour s'exercer, consulter votre serie de cours)

- Si la Transformation est Isentropique, alors il suffit de calculer l'enthalpie à l'entrée, elle sera égale à celle à la sortie. Dans la table thermodynamique, on projette cette valeur, sinon on fait une interpolation linéaire

- Si la transformation n'est pas isenthalpique, il faut alors consulter la table pour déterminer $h_{1}$ puis à l'aide de l'équation de consevation de l'énergie, on détermine $h_{2} d^{\prime}$ 'où on procède comme indiqué ci-dessus.

- Lors d'un couplage Moteur-Climatiseur, on s'intéresse à la quantité de Chaleur extraite à la source froide et à celle absorbée par le moteur (source onereuse), on écrit le premier principe de la Thermodynamique ainsi que l'égalité de Clausius Clapeyron. 


\section{Diagramme de Mollier}

Un diagramme de Mollier[3] ou appelé également diagramme enthalpique est un diagramme thermodynamique relatif aux fluides, à leur changement d'état (liquide/vapeur) et à leurs pressions/températures d'utilisation.

- L'abscisse correspond à la valeur entropique du point, en $\mathrm{kJ} /(\mathrm{kgK})$.

- L'ordonnée à son enthalpie, en $\mathrm{kJ} / \mathrm{kg}$.

Il doit son nom au physicien et ingénieur allemand Richard Mollier (1863-1935) qui l'a proposé en 1904[3][6].

Ce diagramme sert à determiner la quantité de chaleur produite ou consommée par une variation de température et/ou d'état d'un fluide. Les Frigoristes utilisent des diagrammes enthalpiques pour y traçer le cycle parcouru par le fluide fririgène dans une machine.

Attention: Le diagramme de mollier nous indique une pression absolue et non pas une pression relative, cette dérniére est lue sur le manométre des frigoristes.

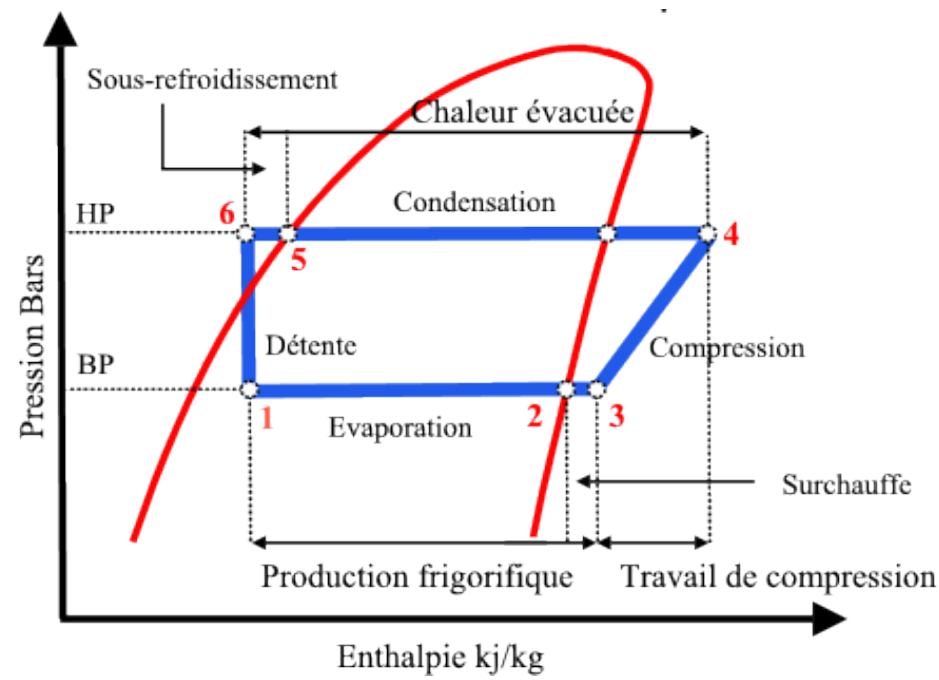

Figure 9. Diagramme de Mollier d'un fluide réfrigérant

\section{L'Isotherme}

- Verticale dans la zone liquide

- Horizentale dans la zone mélange liquide + vapeur

- Incurvée dans le domaine vapeur

L'Isentrope n'est pas représentée dans le domaine vapeur, elle caractérise la compression.

L'Isochore Représentée dans le domaine mélange liquide + vapeur

Elle indique le volume massique et donc le débit volumique dans le compresseur.

Iso-Teneur ou Isotitre Ce sont les courbes à pourcentages de vapeur dans le mélange constant. Varient de 0 à 1.

Fluide Pur ou mélange azéotrope

Ils changent de phases à pression et température constantes, le mélange azéotrope est un liquide qui bout à température constante en gardant une composition fixe.

Fluide zéotrope

C'est un mélange liquide qui bout à température variable en perdant sa composition fixe. Il change d'état à pression constante sous un écart de température qu'on appelle Glissement (Glide) noté GL telle que:

$$
G L=T_{R}-T_{B}
$$

avec $T_{R}$ est la température de rosée, c'est la température à laquelle disparaît la derniére goutte de liquide

$T_{B}$ est la température à laquelle apparaît la premiére bulle de vapeur. 
Le cycle Frigorifique comporte 4 étapes:

(Le Fonctionnement d'une machine frigorifique sera détaillé par la suite)

- L'évaporation: se fait à basse température

- La Compression: se fait dans le Compresseur

- La Condensation: se fait à haute température

- La Détente: se fait dans le Détendeur (Isenthalpique)

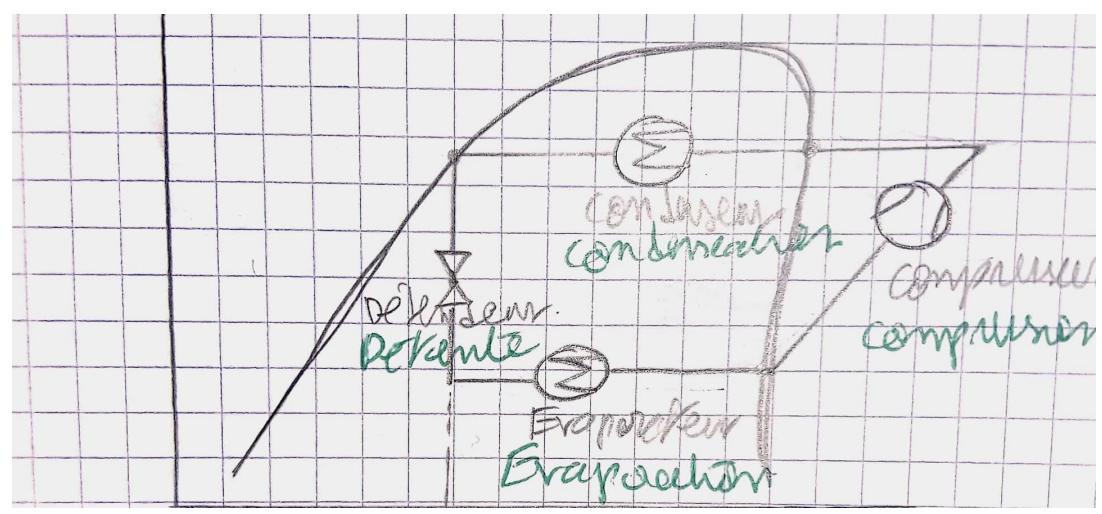

Figure 10. Le Principe d'un cycle Frigorifique

\subsection{Codification Universelle}

Rxyz c'est la codification des fluides réfrigérants

- $\quad \mathrm{R}$ pour dire fluide réfrigérant

- $\quad x$ c'est le nombre d'atomes de carbone - 1

- $\quad$ y c'est le nombre d'atomes d'Hydrogéne + 1

- $\quad$ z étant le nombre d'atomes de Fluor

Remarque: la valence du carbone se compléte avec l'ajout du Chlore Cl.

Si $x=0, x$ est omis

Les isoméres de position des dérivés du propane ont 2 lettres miniscules qui suivent le numéro.

La premiére lettre indique le groupe d'atome:

- a- $\mathrm{CCl}_{2}$

- b- $\mathrm{CClF}$

- $\quad \mathrm{c}-\mathrm{CF}_{2}$

- d- $\mathrm{CClH}$

- e- $\mathrm{CFH}$

- $\mathrm{f}-\mathrm{CH}_{2}$

La Deuxième lettre caractérise la symétrie du composé en faisant la différence des masses moléculaires du premier et et Troisiéme carbone et à la différence la plus faible correspond le composé le plus symétrique.

\section{Alcènes et leurs dérviés halogénés:}

de la même maniére que les alcanes en ajoutant "1" avant le chiffre des centaines; R1xyz Hydrocarbures cycliques et leurs dérivés:

de la même maniére que les alcanes en ajoutant "c" : Rcxyz

Composés Organiques divers:

Constituant la serie 600, par exemple:

-R600: butanes et isoméres

-R610: composés oxygénés

Composés inorganiques divers:

Constituant la serie 700 et le numéro d'identification est obtenu en sommant la masse moléculaire +700 : par exemple:

-Ammoniac (y compris l'ammoniaque): R717, -Eau: R718 
Mélanges Zéotropiques:

Constituent la serie 400

Mélanges Aézeotropiques:

Constituent la serie 500

Remarque: Notons que certaines sociétés chimiques peuvent adopter au lieu de la lettre " $R$ ", le nom du liquide, tels que pour le nom d'une famille de gaz hydrochlorofluorocarbonés (HCFC) ou Chlorofluorécarbonés (CFC), on trouve: Fréon, Forane, Frigen, Solkane, Kléa...

\subsection{Machine Frigorifique}

La Machine Frigorifique extrait de la chaleur $\left(Q_{f}>0\right)$ à la source froide pour la refroidir. Elle reçoit un travail $(W>0)$ et cède une quantité de chaleur $\left(Q_{c}<0\right)$ à la source chaude.

Le transfert d'énergie se fait au moyen d'un fluide frigorigène qui subit une succession de transformations.

- COMPRESSION: Les vapeurs du fluide frigorigène arrivent au niveau du compresseur à basse pression, le fluide sera comprimé, la pression et la température croissent.

- CONDENSATION: le fluide frigorigène arrivant à haute pression, il cède une partie de ses calories à la source chaude et se condense.

-Désurchauffe jusqu'à la température de condensation $T_{K}$

-Condensation

-Sous refroidissement

- DETENTE: le fluide passe de l'état liquide à haute pression vers l'état liquide + vapeur à basse pression, c'est à dire qu'il y aura une vaporisation partielle du fluide.

- EVAPORATION: Après détente, le mélange (liq + vap) passe dans l'évaporateur.

Le liquide restant du mélange s'évapore à pression constante en puisant l'énergie nécessaire dans la source froide.

Les vapeurs sont surchaufées pour éviter au compresseur de fonctionner dans le régime humide, pour éviter les coups de liquide.

Remarque:

Si la compression n'est pas isentropique, le point sera à l'intersection de l'isobare HP et la température T.

La Détente est isenthalpique.

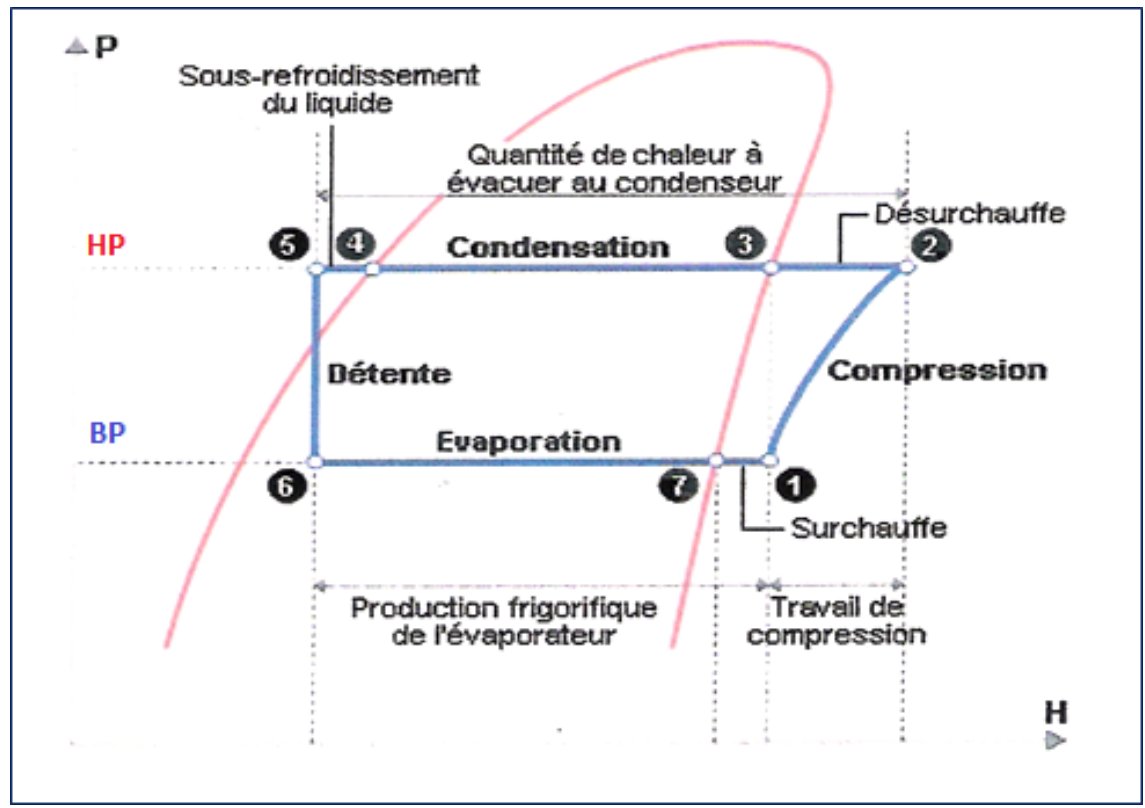

Figure 11. Déscription détaillée d'un cycle Frigorifique 
Production Frigorifique Elle se fait au niveau de l'évaporateur

$$
Q_{f}=h_{1}-h_{6}
$$

textbfProduction Calorifique Elle se fait au niveau du Condenseur

$$
Q_{c}=h_{2}-h_{5}
$$

Remarque: Au niveau du condenseur, on évacue de la chaleur, donc selon la convention des signes, elle sera comptablilisée négative

\subsection{Compresseur Alternatif à piston}

Un compresseur alternatif ou compresseur à pistons est un compresseur mécanique qui sert à fournir des gaz à haute pression. Pour y parvenir, il a recours à des pistons entraînés par un vilebrequin[7][8].

Le vilebrequin est un dispositif mécanique qui permet, par l'intermédiaire d'une bielle, la transformation du mouvement linéaire rectiligne du piston en un mouvement de rotation continu, et inversement.

La culasse est la pièce assurant la fermeture haute du ou des cylindres et, regroupant certaines fonctions d'un moteur à pistons alternatifs. Sur de nombreux types de moteurs, les soupapes d'admission et d'échappement y sont logées. Sa forme et ses caractéristiques sont toujours étroitement liées à l'évolution des moteurs et, plus particulièrement déterminée par le type de distribution et, par la forme de la chambre de combustion[9].

Si le compresseur est à un seul cylindre, alors il est monoétagé

PMH: Le point géographique haut du cylindre; le point où le piston inverse sa course ascendante.

PMB: Le point géographique Bas du cylindre, le point où le piston inverse sa course déscendante.

\subsubsection{Déscription Générale du fonctionnement}

les gaz à l'entrée sont aspirés par une tubulure d'admission via un clapet antiretour, puis s'écoulent dans le cylindre quand le piston descend. A la remontée de ce dernier, le clapet antiretour d'admission se verouille et clui de la tubulure d'échappement s'ouvre. Le gaz est alors comprimé.

\subsubsection{Déscription détaillée}

le piston initialement au PMH amorce sa course descendante jusqu'au PMB ce qui crée une dépression à l'intérieur du cylindre.

La pression dans la conduite d'aspiration force le clapet d'aspiration à s'ouvrir, le clapet de refoulement étant fermé.

Le piston commence sa course ascendante de compression,comprime le gaz et la pression, en augmentant, ferme le clapet d'admission.

La pression du cylindre augmente et n'est pas encore suffisante pour ouvrir le clapet de refoulement et c'est la seule étape thermodynamique

Quand la pression du fluide dépasse largement la pression de tarage du clapet de refoulement; celui-ci s'ouvre et le gaz comprimé s'échappe.

Le Piston finissant sa remontée,chasse lefluide du cylindre vers la conduite de refoulement. Arrivé au PMH, il reste un éspace entre me piston et la culasse qui n'est jamais balayé: c'est le volume mort: cet espace permet de compenser les jeux mécaniques et l'effet de dilatation des piéces. Au cours de sa course déscendante; me clapet de refoulement se ferme, le gaz à l'intérieur du cylindre se détend, la pression chute et quand elle dépasse légérement la pression d'aspiration, le clapet d'aspiration s'ourve : le gaz remplit le cylindre. Et un nouveau cycle commence... 


\subsubsection{Grandeurs Caractéréstiques}

La Cylindrée: c'est le volume balayé par les pistons lors de leurs courses d'aspirations.

$$
C=\frac{N \pi \varphi^{2} L}{4}
$$

avec

$\mathrm{N}$ : nombre de cylindre

L: la course (PMH-PMB)

$\varphi:$ Alésage $=$ diamétre intérieur du cylindre

\section{Débit Volume balayé}

$$
q_{v b}=\frac{n c}{60}
$$

Débit volume aspiré

c'est le volume réellement aspiré

$$
q_{v a}=\eta_{v} q_{v b}
$$

$\eta_{v}$ est le rendement volumétrque

\section{Débit massique}

$$
q_{m}=\frac{\eta_{b} q_{v b}}{v}
$$

$v$ étant le volume massqiue de la vapeur

\section{Taux de Compression}

$$
\tau=\frac{H P}{B P}
$$

$\tau<10$ pour un seul étage

$\tau=4$ pour les gaz inflammables

si $\tau$ est important, alors une compression sur plusieurs étages est recommendée.

\section{Rendement volumétrique}

$$
\eta_{v}=\frac{q_{v a}}{q_{v b}}
$$

une expression empirique est donée par:

$$
\eta_{v}=1-0.04 \tau
$$

\section{Rendement indiqué}

$$
\eta_{i}=\frac{W_{i s}}{\text { Wrel }}
$$

\section{Rendement mécanique}

$$
\eta_{m}=\frac{W_{\text {rel }}}{\text { Wabsorb }}
$$

Le travail absorbé est appelé aussi le travail effectif.

\section{Rendement Global}

Appelé aussi rendement effectif, il tient compte de tous les écarts par rapport à la compression isentropique

$$
\eta_{g}=\eta_{i}=\frac{W_{i s}}{W_{a}}
$$

une autre expression empirique peut être aussi employée:

$$
\eta_{\text {eff }}=0.8-0.004(\eta-5)^{2}-\frac{0.5}{\tau-0.3}
$$




\section{Rendement Isentropique}

$$
\eta_{i s}=\frac{W_{\text {rel }}}{W_{i s}}
$$

Rendement d'une compression Isotherme

$$
\eta=\frac{q_{m} r T \ln \beta}{P_{\text {relle }}}
$$

Rendement d'une compression adiabatique

$$
\eta=\frac{q_{m} C_{p} T_{1}\left(\beta^{\frac{\gamma-1}{\gamma}}-1\right)}{P_{\text {relle }}}
$$

\subsection{Diagrammes Thermodynamiques}

Diagramme de Clapyeron

C'est le diagramme PV classique.

Loi de la transformation isotherme: $\mathrm{PV}=\mathrm{Cte}$

L'isotherme est une hyperbole.

Les isenthalpes se confondent avec les isothermes les isentropes sont plus pentées que les isothermes

Le diagramme PV permet de déterminer le travail d'un système ouvert ou fermé.

- Cycle fermé:

$$
W=-\int p d v
$$

- Cycle ouvert:

$$
W_{t}=\int v d p
$$

\section{Diagramme Entropique}

C'est le diagramme TS.

Ce diagramme permet de déterminer la quantité de chaleur échangée.

$$
Q=\int T d s
$$

Pour une Transformation isochore:

$$
Q=C_{v} d T
$$

Pour une transformation isobare:

$$
Q=C_{p} d T
$$

L'isochore est au-dessus de l'isobare.

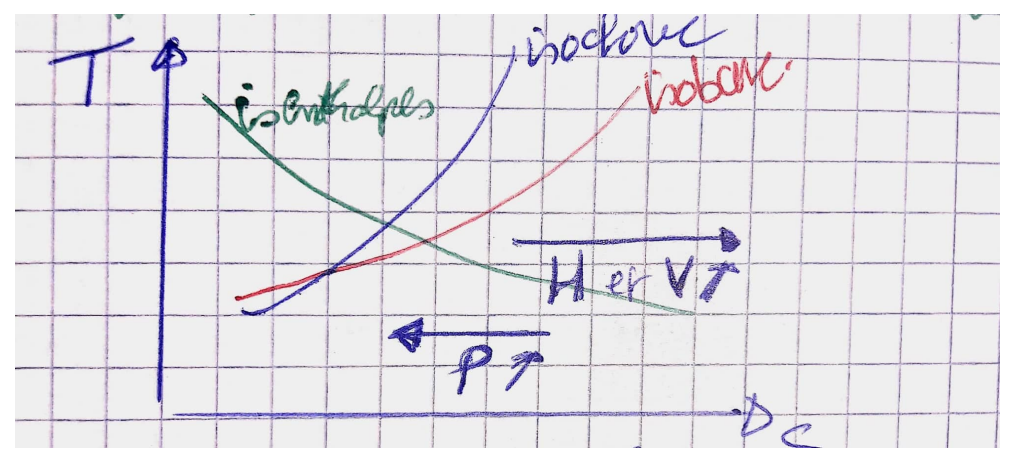

Figure 12. Diagramme Entropique 


\section{Diagramme Enthalpie-Entropie}

Il permet une lecture des enthalpies et entropies de transformations.

Si les gaz sont parfaits: 1 'isotherme est horizentale

Si le gaz n'est pas parfait: l'isotherme n'est pas horizentale.

Ce diagramme permet d'obtenir la valeur du rendement isentropique d'une détente ou d'une compression.

Dans la région vapeur surchauffée, les isobares ont une allure d'exponentielle.

Les isothermes se confondent avec les isenthalpes.

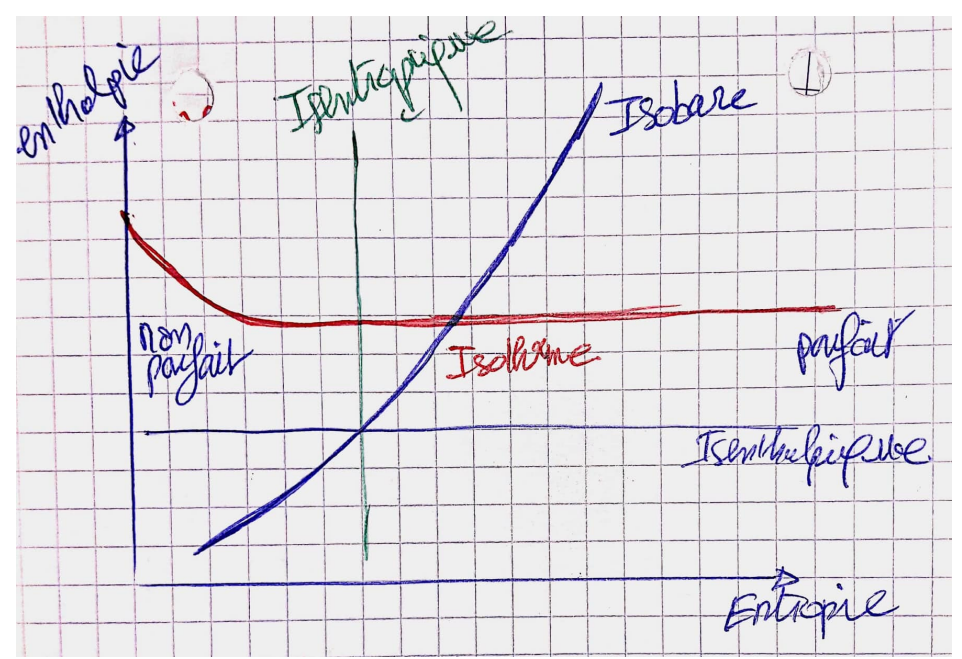

Figure 13. Diagramme Enthalpie-Entropie

Le diagramme enthalpie-entropie s'appelle aussi le diagramme de mollier, dans lequel:

- Les isobares ont une forme exponentielle

- Les isothermes se confondent avec les isenthalpes dès qu'on s'écarte des domaines des hautes pressions.

Qu'est ce qu'un laminage de la vapeur?

Le laminage de la vapeur est un truc dont on entend souvent parler, il consiste simplement à faire brutalement baisser la pression de la vapeur dans une tubulure en réduisant le passage utile du flot par une vanne ou un diaphragme et sans lui demander de produire du travail.

\section{Remarque:}

Attention aux unités!!

$1 \mathrm{~kg} / \mathrm{cm}^{2}=1$ bar

$1 \mathrm{~N} / \mathrm{m}^{2}=0,1$ bar

$1 \mathrm{~Pa}=0.0001 \mathrm{~N} / \mathrm{cm}^{2}$ 
4.5. Rappel de notions de compresseurs

- Compresseur isotherme:

$$
W_{t}=\int_{p_{1}}^{p_{2}}=m r T \ln \frac{P_{2}}{P_{1}}
$$

- Compression isentropique:

$$
W_{t}=\frac{\gamma\left(P_{2} V_{2}-P_{1} V_{1}\right)}{\gamma-1}=m C_{p} T_{1}\left(\beta^{\frac{\gamma-1}{\gamma}}-1\right)
$$

- Compression Polytropique:

$$
W_{t}=\frac{k\left(P_{2} V_{2}-P_{1} V_{1}\right)}{k-1}=\frac{k}{k-1} m r T_{1}\left(\beta^{\frac{k-1}{k}}-1\right)
$$

Astuce de Travail !!

Si on est appelé de calculer le débit d'eau, on détermine le travail (puissance également) de compression polytropique ou isentropique ou isotherme et on applique le premier principe de la thermodynamique à un système ouvert

\subsection{Réfrigération des compresseurs}

La compression isotherme est celle qui consomme le moins du travail, elle est trés difficile à réaliser.

La configuration téchnologique rend la réfrigération peu compétitive en dessous d'un taux de compression de 3.

Il n'ya d'interêt à réfrigérer qu'au delà d'un taux de compression de 4 .

On dispose d'une réfrigération interne et externe:

- Compresseur à réfrigération interne:

Les Surfaces fixes sont aménagées de manière à être parcourues par un fluide de refroidissement.

- Compresseur à réfrigération externe:

Le compresseur est subdivisé en plusieurs corps/étages séparés par des échangeurs.

$$
T_{f}=T_{a} \beta^{\frac{k-1}{k}} \leq 227 C
$$

Si $\beta=4$ ou 5, la comperssion en un seul étage détériore le rendement volumétrique.

- Travail de compression bi-étagée:

$$
W=\frac{k}{k-1} m r T_{1}\left(\left(\frac{P_{i}}{P_{1}}\right)^{\frac{k-1}{k}}-1\right)+\frac{k}{k-1} m r T_{i}\left(\left(\frac{P_{2}}{P_{1}}\right)^{\frac{k-1}{k}}-1\right)
$$

Le travail minimum est obtenu pour:

$\frac{\partial w}{\partial p_{i}}=0$

D'où on aura:

$$
P_{i}=\sqrt{P_{1} P_{2}\left(\frac{T_{i}}{T_{1}}\right)^{\frac{k}{k-1}}}
$$

- Travail de compression multi-étagée

$$
P_{j}=\sqrt{\left(\frac{T_{j}}{T j-1}\right)^{\frac{k}{k-1}} P_{j-1} P_{J+1}}
$$


et si la compression est isotherme:

$$
P_{j}=\sqrt{P_{j-1} P_{j+1}}
$$

Astuces:

Attention!! Si l'eau est diphasée, il est donc pas question d'utiliser une capacité thermique. Pour exprimer le transfert thermique, on utilise l'enthalpie de changement d'état.

Le rendement du cycle de Rankine s'exprime à partir du travail cédée à la turbine et du travail thermique apporté par le générateur de vapeur.

\section{Analyse Thermique des procédés}

La majorité des procédés industriels sont des procédés qui fonctionnent en régime stationnaire et irréversible.

Travail pour un système ouvert

$$
W=\Delta H+T_{\text {ext }} \Delta S_{\text {ext }}
$$

Travail perdu pour un système ouvert

$$
W_{p}=-T_{\text {ext }}\left(\Delta S+\Delta S_{\text {ext }}\right)=-T_{\text {ext }} \Delta S_{\text {ext }}
$$

$W_{p}=0:$ Transformation réversible

$W_{p}<0$ : Transformation réelle

Remarque:

Plus l'irréversibilité est grande, plus la variation d'entropie est grande et plus important sera le travail perdu

\section{Rendement Energétique}

- Turbine:

$$
r=\frac{W_{r e l}}{W_{r v}}
$$

- Compresseur:

$$
r=\frac{W_{r v}}{W_{r e l}}
$$

\section{Exergie}

En thermodynamique, l'exergie est une grandeur permettant de mesurer la qualité d'une énergie. C'est la partie utilisable d'un joule. Le travail maximal récupérable est ainsi égal à l'opposé de la variation d'exergie au cours de la transformation. Un système à l'équilibre thermomécanique ou chimique n'a plus aucune valeur. Plus un système est loin de l'équilibre ambiant, plus il est apte à opérer un changement, aptitude sur laquelle repose l'utilité d'une énergie.

Si selon le premier principe de la thermodynamique la quantité d'énergie se conserve, la qualité de cette énergie, l'exergie, ne peut en revanche que diminuer lors d'une transformation. L'exergie détruite au cours d'une réaction est appelée anergie.

$$
E X=H-T_{\text {ext }} S
$$

Il s'ensuit alors une nouvelle expression du travail:

$$
W_{r e l}=W_{r v}-W_{p}=\Delta E X-E X_{d}
$$

D'où le travail perdu peut être assimilé à une exergie détruite 
6. Thermodynamique des systèmes Ouverts

\subsection{Compression Adiabatique}

Dans le diagramme $\mathrm{h}=\mathrm{f}(\mathrm{s})$, le travail à fournir est minimum si la transformation est réversible.

Si la transformation est irréversible, on a une augmentation de l'entropie et le travail à fournir devient supérieur au travail réversible.

Rendement isentropique:

$$
r_{i s}=\frac{W_{i s}}{W_{r e l}}=\frac{\Delta h_{i s}}{\Delta h_{r e l}}
$$

\subsection{Détente Adiabatique}

Détente sans production de travail

Sans production de travail et sans variation d'énergie potentielle.

$$
\Delta H=\frac{-m \Delta V^{2}}{2}<0
$$

Détente avec production de travail

$$
\Delta H=W
$$

Si la transforamtion est réversible, ce travail sera maximum.

Si la transformation est irréversible, on aura une augmentation d'entropie et le travail produit sera inférieur au travail isentropique.

D'où, le rendement isentropique sera:

$$
r_{i s}=\frac{W_{i s}}{W_{r e l}}
$$

\subsection{Détente de Joule- Thomson}

La détente adiabatique d'un gaz sans production de travail, sans variation d'énergie cinétique et potentielle est appelée Détente de joule-Thomson.

$\Delta H=0$ (détente isenthalpique)

Cette détente se produit avec augmentation d'entropie (Irréversibilité)

Remarque:

-La température d'un fluide est la même avant et aprés laminage

-Si ce n'est pas le cas, alors la vapeur n'est pas un gaz parfait.

\section{Point Bonus}

Chauffage ou changement d'état? Comment procéder?

- $\quad$ Simple Chauffage (ou refroidissement)

$$
Q=m C_{p} \Delta T
$$

- Changement d'état

$$
\begin{gathered}
Q=m L \\
Q=m \Delta H_{\text {changementd'tat }}
\end{gathered}
$$


1 Author Contributions: LABIDI Aymen, please turn to the Research gate and Linkedin for the term 2 explanation and more scientific papers.

3 Institutional Review Board Statement: National Institute of Applied Sciences and Technology,

4 Tunis, Tunisia, Chemical Engineering Student, Chemical Engineering Department

5 Sample Availability: Samples and more researches and papers are available from author'sResearch

6 gate profile

7 Abbreviations

8 The following abbreviations are used in this article:

HP Haute pression

BP Basse Pression

PMH Point Mort Haut

10 PMB Point Mort Bas

PAC Pompe à Chleur

GL Glissement

11 Appendix G Other sources

12 to learn more about chemical engineering, you can search for articles in Techniques de

13 l'ingénieurs

\section{References}

1. P. Trambouze and J.-P. Wauquier, Le développement des procédés de raffinage et de pétrochimie, Éditions Technip, 1975.

2. R. Solimando, L. Schuffenecker and J.-N. Jaubert, Propriétés thermodynamiques du corps pur, Ed. Techniques Ingénieur, 2000.

3. P. Febvre, R. Taillet and L. Villain, Dictionnaire de physique, De Boeck Superieur, 2013.

4. E. Josse, Expériences récentes sur les machines thermiques de basse température dites machines-abwaerme, Walter de Gruyter GmbH \& Co KG, 2019.

5. R. Boyer et al., Evolution des modèles productifs et hybridation: Géographie, histoire et théorie, CEPREMAP, 1997.

6. F. Gugelot, Archives de sciences sociales des religions, 2015, 334-334.

7. H. P. Bloch and J. J. Hoefner, Reciprocating compressors:: operation and maintenance, Elsevier, 1996.

8. J. Townsend, M. A. Badar and J. Szekerces, Engineering science and technology, an international journal, 2016, 19, 566-573.

9. B. SAIFEDDINE, PhD thesis, UNIVERSITE DE MOHAMED BOUDIAF M'SILA FACULTE DE TECHNOLOGIE, 2016. 
